\title{
Evidence for the production of three massive vector bosons with the ATLAS detector
}

\section{Markus Cristinziani*i}

On behalf of the ATLAS Collaboration

Physikalisches Institut, Universität Bonn, Nussallee 12, 53115 Bonn, Germany.

E-mail: cristinz@uni-bonn.de

\begin{abstract}
The search for the production of three massive vector bosons in proton-proton collisions, performed using data at $\sqrt{s}=13 \mathrm{TeV}$ recorded with the ATLAS detector at the Large Hadron Collider in the years 2015-2017, corresponding to an integrated luminosity of $79.8 \mathrm{fb}^{-1}$, is presented. Events with two same-sign leptons $\ell$ (electrons or muons) and at least two reconstructed jets are selected to search for $W W W \rightarrow \ell v \ell v q q$. Events with three leptons without any same-flavour opposite-sign lepton pairs are used to search for $W W W \rightarrow \ell v \ell v \ell v$, while events with three leptons and at least one same-flavour opposite-sign lepton pair and one or more reconstructed jets are used to search for $W W Z \rightarrow \ell v q q \ell \ell$. Finally, events with four leptons are analysed to search for $W W Z \rightarrow \ell v \ell v \ell \ell$ and $W Z Z \rightarrow q q \ell \ell \ell \ell$. Evidence for the joint production of three massive vector bosons is observed with a significance of 4.1 standard deviations, where the expectation is 3.1 standard deviations.
\end{abstract}

7th Annual Conference on Large Hadron Collider Physics — LHCP2019

20-25 May, 2019

Puebla, Mexico

\footnotetext{
*Speaker.

${ }^{\dagger}$ Supported by the European Research Council grant ERC-CoG-617185 and by the German Federal Ministry of Education and Research (FSP-103)
} 


\section{Introduction}

The joint production of three vector bosons is a rare process in the Standard Model (SM). Studies of triboson production can test the non-Abelian gauge structure of the SM theory and any deviations from the SM prediction would provide hints of new physics at higher energy scales. Triboson production has been studied at the Large Hadron Collider (LHC) using proton-proton $(p p)$ collision data taken at $\sqrt{s}=8 \mathrm{TeV}$ for processes involving at least one photon, and for the $W W W$ process [1].

Here, the first evidence for the joint production of three massive vector bosons in $p p$ collisions using the dataset collected with the ATLAS detector [2] between 2015 and 2017 at $\sqrt{s}=13 \mathrm{TeV}$ [3], for a total integrated luminosity of $79.8 \mathrm{fb}^{-1}$, is presented. At leading order (LO) in QCD, the production of three massive vector bosons $(V V V$, with $V=W, Z)$ can proceed via the radiation of each vector boson from a fermion, from an associated boson production with an intermediate boson $\left(W, Z / \gamma^{*}\right.$ or $\left.H\right)$ decaying into two vector bosons, or from a quartic gauge coupling vertex. Representative Feynman diagrams are shown in Figure 1.
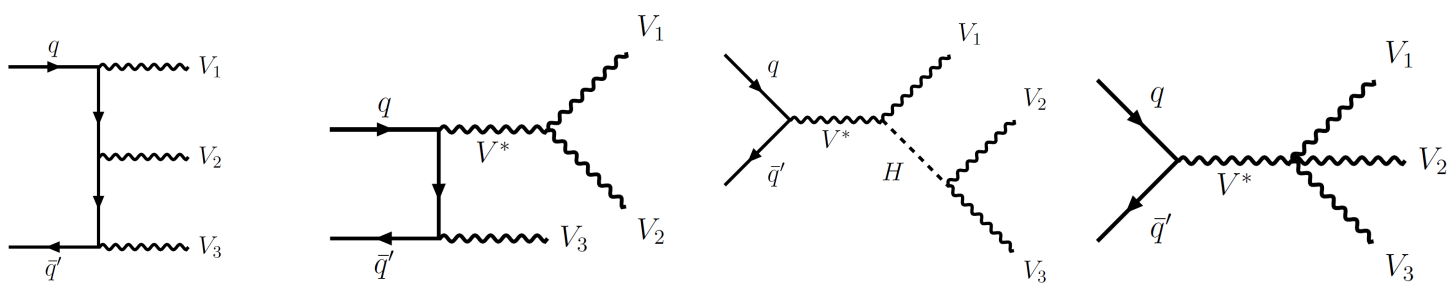

Figure 1: Representative Feynman diagrams at LO for the production of three massive vector bosons, including diagrams sensitive to triple and quartic gauge couplings.

\section{Analysis strategy}

Two dedicated searches are performed, one for the $W^{ \pm} W^{ \pm} W^{\mp}$ (denoted as $W W W$ ) process and one for the $W^{ \pm} W^{\mp} Z$ (denoted as $W W Z$ ) and $W^{ \pm} Z Z$ (denoted as $W Z Z$ ) processes. To search for the $W W W$ process, events with two same-sign leptons with at least two jets resulting from $W W W \rightarrow \ell v \ell v q q(\ell=e, \mu$, including $\tau \rightarrow \ell v v)$ or three leptons resulting from $W W W \rightarrow \ell v \ell v \ell v$ are considered and are hereafter referred to as the $\ell \nu \ell v q q$ and $\ell v \ell v \ell v$ channels, respectively. To search for the $W W Z$ and $W Z Z$ (denoted as $W V Z$ ) processes, events with three or four leptons resulting from $W V Z \rightarrow \ell v q q \ell \ell, W W Z \rightarrow \ell v \ell v \ell \ell$, and $W Z Z \rightarrow q q \ell \ell \ell \ell$ are used. Selection criteria are chosen in order to ensure there is no overlap between different channels. A discriminant that separates the $W W W$ or $W V Z$ signal from the background is defined in each channel. The discriminants are combined using a binned maximum-likelihood fit, which allows the signal yield and the background normalisations to be extracted. The combined observable is the signal strength parameter $\mu$ defined as the ratio of the measured $W V V$ cross section to its SM expectation, where one common ratio is assumed for $W W W$ and $W V Z$.

Signal and background processes were simulated with several Monte Carlo (MC) event generators. Triboson signal events [4] were generated using SHERPA, where all three bosons are on-massshell, using a factorised approach [5]. Events with an off-mass-shell boson through $W H \rightarrow W V V^{*}$ 
and $Z H \rightarrow Z V V^{*}$ were generated using Powheg-Box for the $W W W$ analysis, while for the $W V Z$ analysis PyтніA was used. Both on-mass-shell and off-mass-shell processes were generated at nextto-leading order (NLO) QCD accuracy and are included in the signal definition.

Diboson ( $W W, W Z, Z Z), W / Z+\gamma$ and single boson ( $W / Z+$ jets) production, as well as electroweak production of $W^{ \pm} W^{ \pm}+2$ jets, $W Z+2$ jets, and $Z Z+2$ jets, were modelled using SHERPA. In order to improve the agreement between the simulated and observed jet multiplicity distributions for the $W Z \rightarrow \ell v \ell \ell$ and $Z Z \rightarrow \ell \ell \ell \ell$ events, a jet-multiplicity based reweighting was applied to the simulated $W Z$ and $Z Z$ samples. Top-quark pair events $(t \bar{t})$ and other background processes containing top quarks were generated using PowHEG-Box or MADGRAPH5_aMC@NLO.

Leptons are required to pass certain identification quality requirements and to be isolated from other particles in both the calorimeters and the inner detector. The lepton isolation cone size is at most $\Delta R=0.2$, except for the muon isolation in the inner detector, where it is at most $\Delta R=0.3$. The requirements are more restrictive in the $W W W$ analysis because a larger contamination is expected from jets misidentified as leptons or leptons from hadron decays (including $b$ - and $c$ hadron decays), referred to as "non-prompt" leptons in the following.

A dedicated boosted decision tree (BDT), termed "non-prompt lepton BDT" [6], is used to reject leptons likely to originate from heavy-flavour decays. In addition, electrons have to pass the "charge misidentification suppression BDT" [7] to reject electrons likely to have the electric charge wrongly measured.

\section{Analysis targeting $W W W$}

The experimental signature of the $\ell v \ell v q q$ process is the presence of two same-sign leptons, $E_{\mathrm{T}}^{\mathrm{miss}}$, and two jets. The signature of the $\ell \nu \ell v \ell v$ process is the presence of three leptons and $E_{\mathrm{T}}^{\mathrm{miss}}$. To reduce the background contributions from processes that have more than two (three) leptons in the $\ell v \ell v q q$ ( $\ell \ell v \ell v)$ channel a "veto lepton" definition is introduced. Compared with the nominal lepton selection criteria, the veto lepton $p_{\mathrm{T}}$ threshold is lowered, and the isolation, non-prompt lepton BDT, charge misidentification suppression BDT, and impact parameter requirements are removed.

To select $\ell \ell \ell v q q$ candidates, events are required to have exactly two nominal leptons and the same electric charge, at least two jets, and no identified $b$-jets. Four regions are considered, based on the lepton flavour, namely $e e, e \mu, \mu e$, and $\mu \mu$. The invariant mass of the dilepton system is required to be in the range $40<m_{\ell \ell}<400 \mathrm{GeV}$. The upper mass limit reduces the contribution from the $W Z+$ jets process. A dijet system, formed by the two jets with the largest $p_{\mathrm{T}}$, is required to have $m_{j j}<300 \mathrm{GeV}$ and $\left|\Delta \eta_{j j}\right|<1.5$. The cuts applied on the dijet system mainly reduce the contributions from the same-sign $W W$ vector boson scattering process. Additionally, in the ee final state, $E_{\mathrm{T}}^{\text {miss }}$ is required to be above $55 \mathrm{GeV}$ and $m_{\ell \ell}$ must satisfy $m_{\ell \ell}<80 \mathrm{GeV}$ or $m_{\ell \ell}>100 \mathrm{GeV}$, to reduce contamination from $Z \rightarrow e e$ where the charge of one electron is misidentified. To select $\ell v \ell v \ell v$ candidates, events are required to have exactly three nominal leptons and no identified $b$-jets. To reduce the contribution from the $W Z \rightarrow \ell v \ell \ell$ process, events are required to have no same-flavour opposite-sign (SFOS) lepton pairs.

A major background originates from the $W Z+$ jets $\rightarrow \ell v \ell \ell+$ jets process, contributing to the $\ell v \ell v q q$ channel when one lepton is not reconstructed or identified, or to the $\ell v \ell v \ell v$ channel when 
a $Z$ boson decays into a pair of $\tau$ leptons both of which decay to an electron or muon. Simulation is used to estimate this background. Data and simulation agree in a dedicated validation region. Contributions from SM processes that produce at least one non-prompt lepton are estimated using a data-driven method as described in Ref. [8], where data control regions are scaled by a "fake factor", which is derived from two $t \bar{t}$-enriched regions selected with two or three leptons (no SFOS lepton pairs) and exactly one $b$-jet. Events resulting from the $V \gamma j j$ production can pass the $e e$, $e \mu$ and $\mu e$ signal selection criteria if the photon is misreconstructed as an electron. This contribution (referred to as " $\gamma$ conv.") is evaluated using a data-driven method similar to the non-prompt lepton background evaluation by introducing "photon-like" electrons. The charge misidentification background originates from processes that produce oppositely-charged prompt leptons, where one lepton's charge is misidentified and results in final states with two same-sign leptons. The background is estimated using a data-driven technique.

\section{Analysis targeting $W W Z$ and $W Z Z$}

The experimental signature of the $W V Z \rightarrow \ell v q q \ell \ell, W W Z \rightarrow \ell v \ell v \ell \ell$, and $W Z Z \rightarrow q q \ell \ell \ell \ell$ processes is the presence of three or four charged leptons. In order to increase the signal acceptance, "loose" leptons are defined in addition to nominal leptons, the latter being a subset of the former. Loose leptons have both the isolation and non-prompt lepton BDT requirements removed. In addition, for loose electrons the charge misidentification suppression BDT requirement is removed. Six regions are defined with either three or four loose leptons, sensitive to triboson final states containing $Z$ bosons. Among all possible SFOS lepton pairs, the one with $m_{\ell \ell}$ closest to the $Z$ boson mass is defined as the best $Z$ candidate. In all regions, the presence of such a best $Z$ candidate with $\left|m_{\ell \ell}-91.2 \mathrm{GeV}\right|<10 \mathrm{GeV}$, is required. Furthermore, any SFOS lepton pair combination is required to have a minimum invariant mass of $m_{\ell \ell}>12 \mathrm{GeV}$. Events with $b$-tagged jets are vetoed.

For the three-lepton channel, the lepton which is not part of the best $Z$ candidate is required to be a nominal lepton. The scalar sum of the transverse momenta of all leptons and jets $\left(H_{\mathrm{T}}\right)$ is required to be larger than $200 \mathrm{GeV}$. This significantly reduces the contribution of the $Z \rightarrow$ $\ell \ell$ processes with one additional non-prompt lepton. Three regions are defined according to the number of jets in the event: one jet $(3 \ell-1 \mathrm{j})$, two jets $(3 \ell-2 \mathrm{j})$, and at least three jets $(3 \ell-3 \mathrm{j})$. For the four-lepton channel, the third and fourth leading leptons are required to be nominal leptons. The two leptons which are not part of the best $Z$ candidate definition are required to have opposite charges. These "other leptons" are used to define three regions, depending on whether they are different-flavour ( $4 \ell$-DF), or same-flavour and their mass lies within a window of $10 \mathrm{GeV}$ around the $Z$ boson mass ( $4 \ell$-SF-Z) or their mass is outside this window ( $4 \ell$-SF-noZ).

In each of the six regions the distribution of a dedicated boosted-decision-tree discriminant, separating the $W V Z$ signal from the dominant diboson background, is fed as input to the binned maximum-likelihood fit to extract the signal. For the three-lepton channels, 13, 15, and 12 input variables are used for the $3 \ell-1 \mathrm{j}, 3 \ell-2 \mathrm{j}$, and $3 \ell-3 \mathrm{j}$ final states, respectively, while for the four-lepton channels, six input variables are used for each of the $4 \ell$-DF, $4 \ell$-SF-Z and $4 \ell$-SF-noZ final states.

Due to the required presence of nominal leptons in the three- and four-lepton channels, backgrounds with a $Z$ boson and non-prompt leptons are reduced. The remaining backgrounds are dominated by processes with prompt leptons and thus all backgrounds are estimated using simula- 
tion. The $W Z+$ jets and $Z+$ jets backgrounds are validated in a region defined in the same way as the $3 \ell-1 \mathrm{j}$ region, with the exception that no requirement on $H_{\mathrm{T}}$ is applied, the third-highest- $p_{\mathrm{T}}$ lepton is required to have a small transverse momentum $\left(10 \mathrm{GeV}<p_{\mathrm{T}}<15 \mathrm{GeV}\right)$, and the invariant mass of the three leptons has to be smaller than $150 \mathrm{GeV}$. Data and expectation agree in a dedicated validation region. The $t \bar{t} Z$ background is determined in a region defined like the $3 \ell-3 \mathrm{j}$ region with the exception that no requirement on $H_{\mathrm{T}}$ is applied, and at least four jets are required, of which at least two are $b$-tagged. This region is included as a single-bin control region (CR) in the fit model.

\section{Signal extraction and combination}

The $W W W, W W Z$ and $W Z Z$ regions are combined using the profile likelihood method based on a simultaneous fit to distributions in the signal and background control regions. A total of eleven signal regions are considered: four regions $(e e, e \mu, \mu e$, and $\mu \mu)$ for the $\ell v \ell v q q$ channel, one region ( $\mu e e$ and $e \mu \mu$ combined) for the $\ell v \ell v \ell v$ channel, three regions $(3 \ell-1 \mathrm{j}, 3 \ell-2 \mathrm{j}$, and $3 \ell$ -

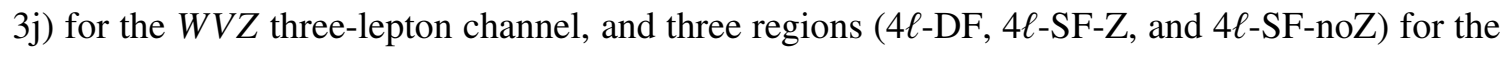
$W V Z$ four-lepton channel. One control region is considered: the $t \bar{t} Z$ control region described in Section 4. The distributions used in the fit are the $m_{j j}$ distributions for the $\ell v \ell v q q$ channel and the BDT distributions for the $W V Z$ three-lepton and four-lepton channels. The number of selected events in the $\ell v \ell v \ell v$ channel and the $t \bar{t} Z$ control region are each included as a single bin in the fit. In total, 186 bins are used in the combined fit. Correlations of systematic uncertainties arising from common sources are maintained across processes and channels.

The simultaneous fit model has the power to constrain the normalisations of the dominant backgrounds from the $W Z$ and $Z Z$ processes at the $\sim 5 \%$ level. The contribution to the $W V V$ signal from $V H$ associated production is $\sim 40 \%$ in the $W W W$ fiducial regions and $\sim 30 \%$ in the $W V Z$ fiducial regions. Figure 2 shows the comparison between data and post-fit prediction of the combined $m_{j j}$ distribution for the $\ell v \ell v q q$ channel, the number of selected events for the $\ell v \ell v \ell v$ channel, and the BDT output distributions in the $3 \ell-2 \mathrm{j}$ and $4 \ell$-DF regions for the $W V Z$ analysis. The $3 \ell-2 \mathrm{j}$ and $4 \ell$-DF regions are chosen since they have the best sensitivity among the three-lepton and four-lepton channels. Data and predictions agree in all distributions.

The overall observed (expected) significance for $W V V$ production is found to be $4.1 \sigma(3.1 \sigma)$, constituting evidence for the production of three massive vector bosons. The combined best-fit signal strength for the $W V V$ process, obtained by the fit to the eleven signal regions and one control region, is $\mu_{W V V}=1.40_{-0.37}^{+0.39}$ with respect to the SM prediction. The statistical uncertainty on the measured signal strength is ${ }_{-0.24}^{+0.25}$ and the systematic uncertainty is ${ }_{-0.27}^{+0.30}$. The largest systematic uncertainties come from uncertainties related to data-driven background evaluations affecting the $W W W$ channels, from theoretical uncertainties related to renormalisation and factorisation scale variations, and from experimental uncertainties.

Fits are also performed separately in the $W W W$ and the $W V Z$ channels. where the other signal strength is fixed to its SM expectation. For the fits of the $W W W$ channels, an additional $W Z$ control region is used in the fit, helping to constrain the overall normalisation of the $W Z+$ jets background, which in the combined fit is constrained by the $W V Z$ three-lepton signal regions. The observed (expected) significance is $3.2 \sigma(2.4 \sigma)$ for $W W W$ production and $3.2 \sigma(2.0 \sigma)$ for $W V Z$ production. 


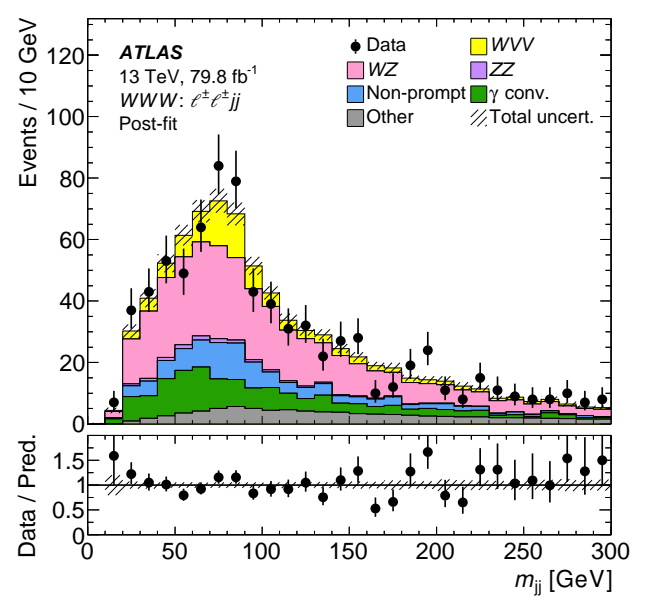

(a)

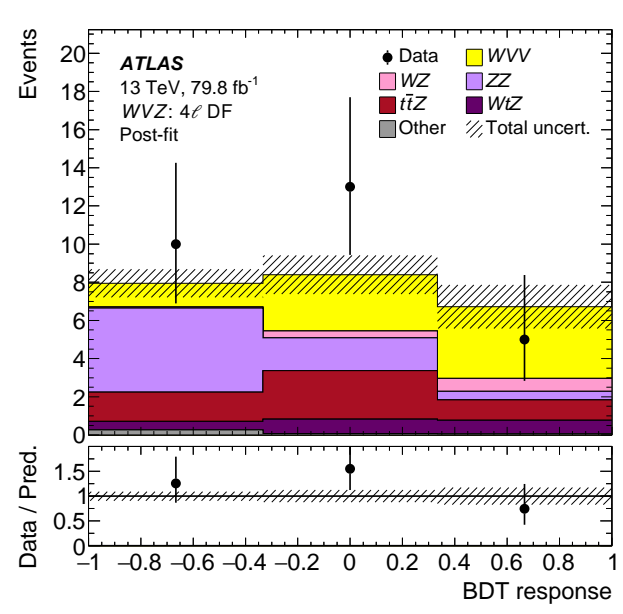

(b)

Figure 2: Post-fit distribution of (a) $m_{j j}$ for the $W W W \rightarrow \ell v \ell v q q$ analysis ( $e e, e \mu, \mu e, \mu \mu$ combined) and (b) the BDT response in the $4 \ell$-DF channel for the $W V Z$ analysis [3]. The uncertainty band includes both statistical and systematic uncertainties as obtained by the fit.

\begin{tabular}{c|cc}
\hline \hline \multirow{2}{*}{ Decay channel } & \multicolumn{2}{|c}{ Significance } \\
& Observed & Expected \\
\hline$W W W$ combined & $3.2 \sigma$ & $2.4 \sigma$ \\
$W W W \rightarrow \ell \nu \ell \nu q q$ & $4.0 \sigma$ & $1.7 \sigma$ \\
$W W W \rightarrow \ell \nu \ell \nu \ell \nu$ & $1.0 \sigma$ & $2.0 \sigma$ \\
\hline$W V Z$ combined & $3.2 \sigma$ & $2.0 \sigma$ \\
$W V Z \rightarrow \ell v q q \ell \ell$ & $0.5 \sigma$ & $1.0 \sigma$ \\
$W V Z \rightarrow \ell v \ell v \ell \ell / q q \ell \ell \ell \ell$ & $3.5 \sigma$ & $1.8 \sigma$ \\
\hline$W V V$ combined & $4.1 \sigma$ & $3.1 \sigma$ \\
\hline \hline
\end{tabular}

Table 1: Observed and expected significances with respect to the SM background-only hypothesis for the four $W V V$ channels entering the fit.

Table 1 and Figure 3(a) summarise the observed and expected significances with respect to the background-only hypothesis and the observed best-fit values of the signal strength for the individual and combined fits. The measured signal strengths from the individual fits are converted to inclusive cross-section measurements using the signal samples and the central values of the theoretical predictions. All uncertainties determined in the fit are included in the conversion, except for the normalisation uncertainty in the signal prediction. The results are: $\sigma_{W W W}=0.65_{-0.15}^{+0.16}$ (stat.) ${ }_{-0.14}^{+0.16}$ (syst.) $\mathrm{pb}$ and $\sigma_{W W Z}=0.55 \pm 0.14$ (stat.) ${ }_{-0.13}^{+0.15}$ (syst.) pb. For the $\sigma_{W W Z}$ extraction, the $W Z Z$ normalisation is fixed to the SM expectation. The cross section of the latter is not reported, since there is not enough sensitivity to this channel to quote a separate cross-section value.

Figure 3(b) shows the data, background and signal yields, where the discriminant bins in all signal regions are combined into bins of $\log _{10}(S / B), S$ being the expected signal yield and $B$ 


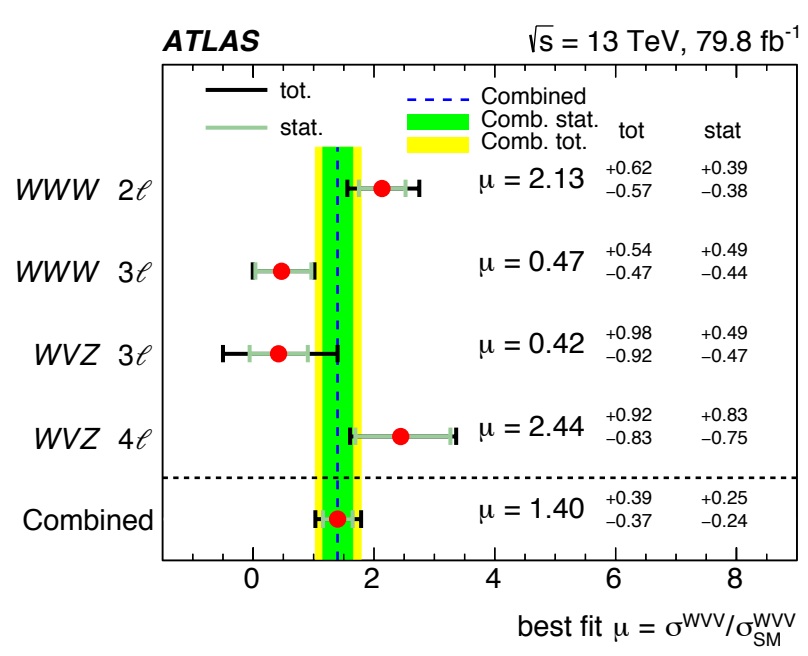

(a)

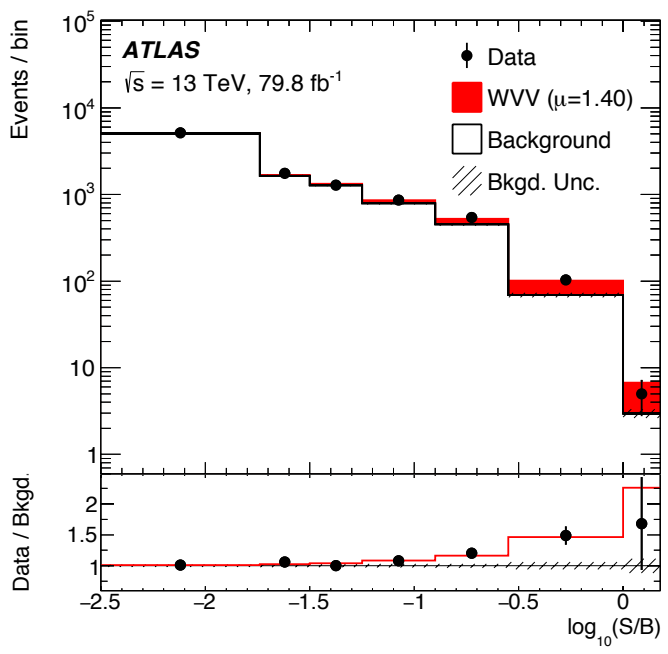

(b)

Figure 3: (a) Extracted signal strengths $\mu$ for the four analysis regions and for the combination. (b) Event yields as a function of $\log _{10}(S / B)$ for data, background $B$ and the signal $S$. Events in all eleven signal regions are included. The background and signal yields are shown after the global signal-plus-background fit. The hatched band corresponds to the systematic uncertainties, and the statistical uncertainties are represented by the error bars on the data points. The lower panel shows the ratio of the data to the expected background estimated from the fit, compared to the expected distribution including the signal (red line) [3].

the background yield. The background and signal yields are shown after the global signal-plusbackground fit to the data.

\section{Summary}

The search for the joint production of three massive vector bosons ( $W$ or $Z$ ) in proton-proton collisions using $79.8 \mathrm{fb}^{-1}$ of data at $\sqrt{s}=13 \mathrm{TeV}$ collected by the ATLAS detector at the LHC, was presented. Events with two, three or four reconstructed electrons and muons were analysed. First evidence for the production of three massive vector bosons has been observed with a combined significance of 4.1 standard deviations, where the expectation is 3.1 standard deviations. The measured production cross sections are $\sigma_{W W W}=0.65_{-0.21}^{+0.23} \mathrm{pb}$, and $\sigma_{W W Z}=0.55_{-0.19}^{+0.21} \mathrm{pb}$, in agreement with the Standard Model predictions.

\section{Acknowledgements}

This work was partially funded by the European Research Council under the European Union's Seventh Framework Programme ERC Consolidator Grant Agreement n. 617185 (TopCoup) and by the German Federal Ministry of Education and Research (BMBF) in FSP-103 under grant n. 05H15PDCAA. 


\section{References}

[1] ATLAS Collaboration, Search for triboson $W^{ \pm} W^{ \pm} W^{\mp}$ production in pp collisions at $\sqrt{s}=8 \mathrm{TeV}$ with the ATLAS detector, Eur. Phys. J. C 77 (2017) 141, arXiv:1610.05088 [hep-ex].

[2] ATLAS Collaboration, The ATLAS Experiment at the CERN Large Hadron Collider, JINST 3 (2008) S08003.

[3] ATLAS Collaboration, Evidence for the production of three massive vector bosons with the ATLAS detector, submitted to Phys. Lett. B, arXiv:1903.10415 [hep-ex].

[4] ATLAS Collaboration, Multi-Boson Simulation for 13 TeV ATLAS Analyses, ATLAS-PHYS-PUB-2017-005, https://cds.cern.ch/record/2261933.

[5] ATLAS Collaboration, Multi-Boson Simulation for 13 TeV ATLAS Analyses, ATLAS-PHYS-PUB-2016-002, https://cds.cern.ch/record/2119986.

[6] ATLAS Collaboration, Evidence for the associated production of the Higgs boson and a top quark pair with the ATLAS detector, Phys. Rev. D 97 (2018) 072003, arXiv:1712.08891 [hep-ex].

[7] ATLAS Collaboration, Electron reconstruction and identification in the ATLAS experiment using the 2015 and 2016 LHC proton-proton collision data at $\sqrt{s}=13 \mathrm{TeV}$, Eur. Phys. J. C 79 (2019) 639, arXiv:1902.04655 [hep-ex].

[8] ATLAS Collaboration, Measurement of $W^{ \pm} W^{ \pm}$vector-boson scattering and limits on anomalous quartic gauge couplings with the ATLAS detector, Phys. Rev. D 96 (2017) 012007, arXiv:1611.02428 [hep-ex]. 